\title{
Medical Treatment of Gastrointestinal Neuroendocrine Neoplasms
}

\author{
Authors \\ Marina Tsoli ${ }^{1}$, Krystallenia Alexandraki ${ }^{1}$, Christos Xanthopoulos ${ }^{1}$, Eva Kassi ${ }^{1},{ }^{2}$, Gregory Kaltsas ${ }^{1}$
}

\author{
Affiliations \\ 1 1st Department of Propaedeutic and Internal Medicine, \\ National and Kapodistrian University of Athens, Athens, \\ Greece \\ 2 Department of Biological Chemistry, National and \\ Kapodistrian University of Athens, Athens, Greece
}

Key words

neuroendocrine tumour, somatostatin analogues, radionuclides, targeted treatment, carcinoid syndrome

$\begin{array}{ll}\text { received } & 01.07 .2019 \\ \text { accepted } & 22.01 .2020\end{array}$

Bibliography

DOI https://doi.org/10.1055/a-1110-7251

Published online: 27.2 .2020

Horm Metab Res 2020; 52: 614-620

(c) Georg Thieme Verlag KG Stuttgart · New York ISSN 0018-5043

Correspondence:

Marina Tsoli MD

1st Department of Propaedeutic and Internal Medicine Laiko Hospital National and Kapodistrian University of Athens

Agiou Thoma

11527 Athens

Greece

Tel.: + 302107462217 , Fax: + 302107462664

martso.mt@gmail.com

\section{ABSTRACT}

Neuroendocrine neoplasms (NENs) are rare tumours that arise mainly in the gastrointestinal or pulmonary system. Most NENs are well-differentiated and may obtain prolonged survival besides the presence of metastatic disease; however, a subset (poorly differentiated NENs) may display a truly aggressive behaviour exhibiting a poor prognosis. The recently developed classification systems along with advances in functional imaging have helped stratify patients to the administration of appropriate therapeutic options. Surgery is the mainstay of treatment of NENs, but in recent decades there has been a considerable evolution of medical treatments that are used for locally advanced or metastatic disease not amenable to surgical resection. Long acting somatostatin analogues are the main therapeutic modality for patients with functioning and well-differentiated low grade NENs exhibiting symptomatic control and mainly stabilisation of tumour growth. Other systemic treatments include chemotherapy, molecular targeted agents, interferon- $\alpha$, peptide receptor radionuclide therapy (PRRT), and immunotherapy. In addition, new agents such as telotristat may be used for the control of symptoms of carcinoid syndrome. The choice and/or sequence of therapeutic agents should be individualized according to tumour origin and differentiation, disease burden, presence of clinical symptoms and patients' performance status in the context of a multidisciplinary approach. Recent advances in the molecular pathogenesis of NENs set the field for a more personalised treatment approach.

\section{Introduction}

Neuroendocrine neoplasms (NENs) constitute a rare group of tumours with an estimated annual incidence of approximately 3-5 cases/100 000 inhabitants [1,2]. NENs are predominately developed in the gastrointestinal (GI) or bronchopulmonary systems but may rarely arise in other sites such as the ovaries and/or the urinary bladder [3]. A subset of these tumours can secrete bioactive compounds leading to distinct clinical syndromes (functioning NENs) or cause symptoms due to mass effects to surrounding structures or through metastatic deposits (non-functioning tumours) [4, 5]. According to the proliferative index (PI) Ki-67, defined by immunohistochemical staining for nuclear Ki-67 protein expression, NENs are classified into grade 1 (G1) if $\mathrm{Ki67} \mathrm{PI}$ is $\leq 2 \%$ or 2 (G2) if Ki-67 PI is between 3 and $20 \%$ and grade 3 (G3) if $\mathrm{Ki}-67 \mathrm{Pl}$ is > $20 \%[6,7]$. However, it subsequently became apparent that the natural histo- ry and prognosis of G3 NENs varied significantly according to their morphological features and subsequent differentiation. Hence, the proposed 2017 WHO classification initially addressing pancreatic NENs (pNENs), divided G3 neoplasms into well-differentiated G3 NEN (defined as G3 NETs) or poorly differentiated G3 neuroendocrine carcinomas (defined as G3 NECs). This classification proved to be valid as it was found to correlate with response to specific treatments and overall prognosis [7]. The recent WHO classification of 2019 has implemented this sub-classification of G3 tumours to all gastro-entero-pancreatic NENs [8].

The management of NENs aims at controlling symptoms related to hormonal hypersecretion along tumour growth and related morbidity and mortality. Surgical resection of the primary tumour and when possible of metastatic involvement is the mainstary of treatment. However, in the presence of extensive disease not amenable to surgical resection several medical therapies are used, 
either alone or in combination, aiming at controlling tumour growth and imroving the symptoms attributed to hormonal excess $[5,9,10]$. In addition, functioning neoplasms may be associated with acute or chronic complications such as carcinoid crisis or carcinoid heart disease that need to be promptly diagnosed and treated $[4,9]$. A number of parameters need to be considered in order to select the most appropriate therapeutic modality in the context of a multidisciplinary approach [11] ( Table 1). The last decade a number of phase III studies have provided good quality data regarding the efficacy of treatments used for GEP-NENs that have set up the field for their medical management ( $\triangleright$ Fig. 1).

\section{Somatostatin analogues (SSAs)}

Somatostatin is a neuropeptide secreted in the gastrointestinal tract and the brain. It acts via interaction with five somatostatin receptor subtypes (SSTR1-5) and inhibits the secretion of various hormones while it may also exert immunomodulatory, cytotoxic and apoptotic actions $[12,13]$. SSTR2 is found predominately in NENs of gastro-entero-pancreatic origin [14].

The somatostatin analogue octreotide was the first that became available and was shown to be associated with significant clinical improvement when administered subcutaneously three times daily in controlling the symptoms of carcinoid syndrome (CS) [15]. Long acting SSAs formulations, octreotide LAR and lanreotide Autogel are currently available and are administered once monthly. These compounds have been shown to be equally effective in the management of symptoms associated with functioning GEP-NENs. In-

- Table 1 Factors determining the therapeutic choice of NENs

\begin{tabular}{|l|l|l|}
\hline Primary tumour & \\
\hline TNM stage & \\
\hline Tumour grade & \begin{tabular}{l} 
Therapeutic decision by \\
a multidisciplinary team \\
Functionality \\
\hline Tumour growth rate \\
\hline Patient's performance status \\
\hline Presence of a familial syndrome \\
\hline Local availability \\
\hline Patient's preference
\end{tabular} \\
\hline
\end{tabular}

deed, control of flushing and diarrhea related to the CS has been reported in 74.2 and $67 \%$ of cases treated with octreotide LAR and lanreotide Autogel, respectively [16].

However, long acting SSAs can also be used for their antiproliferative effect although the molecular basis for their antitumour effect is largely unknown [17]. PROMID was the first randomized placebo-controlled phase III study that showed a significantly prolonged time to progression (TTP) in patients with well differentiated locally advanced or metastatic intestinal NENs on treatment with octreotide LAR [hazard ratio $(H R)=0.34 ; 95 \%$ confidence interval $(\mathrm{Cl})$, 0.20-0.59; $p=0.000072$ ] [18]. Subsequently, CLARINET study, another randomized placebo-controlled phase III trial, demonstrated significantly increased progression free survival (PFS) in patients with various GEP-NENs treated with lanreotide compared to placebo $(\mathrm{HR}=0.47 ; 95 \% \mathrm{Cl}, 0.3-0.73)$ [19]. Thus, SSAs are considered as first line systemic therapy for tumour growth control in cases of stable or slowly progressive disease. Although there is no established Ki-67 cut off value, SSAs are generally recommended in patients suffering from NENs with a Ki-67 value up to $10 \%$ [9]. In selected cases of uncontrolled CS despite adequate treatment with SSAs, decreasing the interval of administration or administration of higher than the labelled doses are associated with higher efficacy [20, 21].

Pasireotide (SOM230), a new synthetic analogue displaying high affinity for all SSTRs except for SSTR4, has also been shown to be efficacious in patients with advanced NENs but recent studies showed no advantage of this agent compared to other SSAs $[22,23]$.

Common adverse events of SSAs are abdominal pain, diarrhea, nausea, gallstone development and glucose intolerance [12]. Pasireotide is a potent hyperglycaemic agent associated with alterations of glucose metabolism in up to $25 \%$ of patients treated $[22,24]$.

\section{Targeted agents}

Molecular studies have revealed that two main pathways are involved on neuroendocrine tumour growth, the vascular endothelial growth factor (VEGF) and the mammalian target of rapamycin (mTOR) pathway and drugs that target these pathways have been proved to be effective in patients with advanced GEP-NENs.

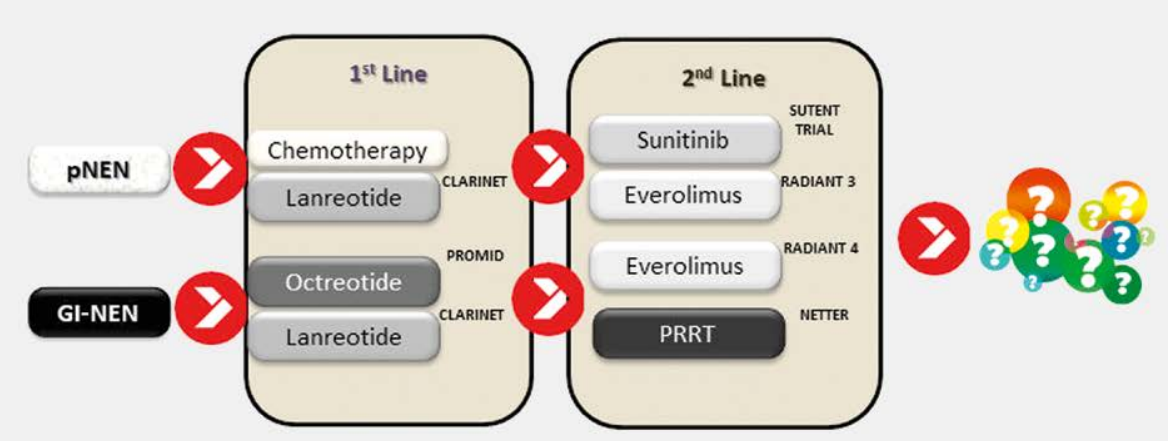

- Fig. 1 Evidence-based (phase III studies except chemotherapy) therapeutic modalities for NENs. pNEN: Pancreatic neuroendocrine neoplasm; GI-NEN: Gastrointestinal neoplasm; PRRT: Peptide receptor radionuclide therapy. 
Everolimus is an mTOR inhibitor that has demonstrated anti-proliferative action in different NENs. The RADIANT trial program that involved a phase II and three randomized phase III trials has shown significant prolongation of PFS with everolimus versus placebo in patients with advanced $G 1$ or $G 2$ pancreatic and intestinal NENs [25-28]. Therefore, everolimus is recommended as a second or third-line treatment after failure of SSAs or other agents mainly in well-differentiated NENs. In addition, a recent meta-analysis reported a significantly high efficacy of the combination treatment of SSAs with everolimus [10]. Careful observation is warranted as everolimus is associated with potential adverse events such as glucose intolerance or diabetes, stomatitis and pneumonitis [29].

The tyrosine kinase inhibitor (TKI) sunitinib inhibits various kinases including the VEGF receptor and displays an anti-angiogenic effect. A randomized phase III study including patients with progressive pNENs has shown significant prolongation of PFS and increased overall survival (OS) after treatment with sunitinib compared to placebo (HR $=0.42 ; 95 \% \mathrm{Cl}: 0.26-0.66 ; \mathrm{p}<0.001)$ [30]. Common adverse events include nausea, diarrhea, fatigue, neutropenia, hypertension, hypothyroidism and palmar-plantar erythrodysesthesia [24]. There are not enough published data regarding treatment with sunitinib in non-pancreatic NENs. Pazopanib and axitinib, that are multi-targeted TKI and bevacizumab, a mono-clonal anti-VEGF antibody have been shown to be effective in NENs but further studies are required to establish their use in clinical practice [31-33].

A recent study showed that there was no significant difference in PFS and OS among patients who received sequential treatment with both everolimus and sunitinib irrespectively of the order of administration while the majority of patients tolerated treatment relatively well [34].

\section{Chemotherapy}

Before the introduction of molecular targeted agents, chemotherapy was the main option of systemic treatment of NENs. Streptozotocin based chemotherapy in combination with 5 -fluorouracil or doxorubicin was shown to be effective in the treatment of pNENs and especially in $\mathrm{G} 2$ neoplasms, and in those with relatively rapid growth or high tumour load [35-37]. However, other well differentiated GI-NENs did not respond well to systemic chemotherapy, while in G3 NECs platinum-based chemotherapy is considered as a first-line option [36, 38-40]. Recent studies have shown that temozolomide as monotherapy or in combination with capecitabine may be an effective treatment option in advanced NENs from various anatomical sites with tolerable toxicity exhibiting response rates in up to $30-40 \%$ of patients ( $\mathbf{F i g}$. 2 ) [41, 42].

In clinical practice, systemic chemotherapy is recommended in G1 or G2 NENs with a high tumour load or displaying significant tumour progression in less than 6-12 months and in G3 NENs. In G3 NECs platinum based chemotherapy is the first line treatment while FOLFOX (folinic acid, 5-fluorouracil, oxaliplatin) or FOLFIRI (folinic acid, 5-fluorouracil, irinotecan) are used as second line treatments $[40,43]$. Systemic chemotherapy in small bowel NENs is not as efficacious as in pNENs except in cases of poorly differentiated tumours albeit the responses obtained are moderate and usually of short duration $[29,44]$.

\section{Peptide Receptor Radionuclide Therapy (PRRT)}

As the majority of GEP-NENs express somatostatin receptors, radiolabelled somatostatin analogues have traditionally been used for tumour diagnosis and recently they have been introduced as a therapeutic option for NENs. A phase III randomized controlled trial (NETTER-1) showed that ${ }^{177}$ Lu-DOTATATE was more effective than high dose octreotide LAR (60 mg) and was associated with significant prolongation of PFS and symptomatic improvement in patients with advanced progressive SSTR-positive intestinal NENs $(\mathrm{HR}=0.21 ; 95 \% \mathrm{Cl}, 0.13$ to $0.33 ; \mathrm{p}<0.001)[29,45]$. A recent meta-analysis showed also that the combination treatment of PRRT with SSAs is considered significantly effective [10]. In addition, PRRT is associated with a substantial improvement of quality of life in patients with progressive midgut NENs $[10,46]$. Currently, there is no established indication for treatment of pNENs with PRRT but it is generally recommended in case of failure of other therapeutic options [29].

It has been recently observed that patients with negative fluorodeoxyglucose PET imaging (FDG-PET/CT) displayed better re-
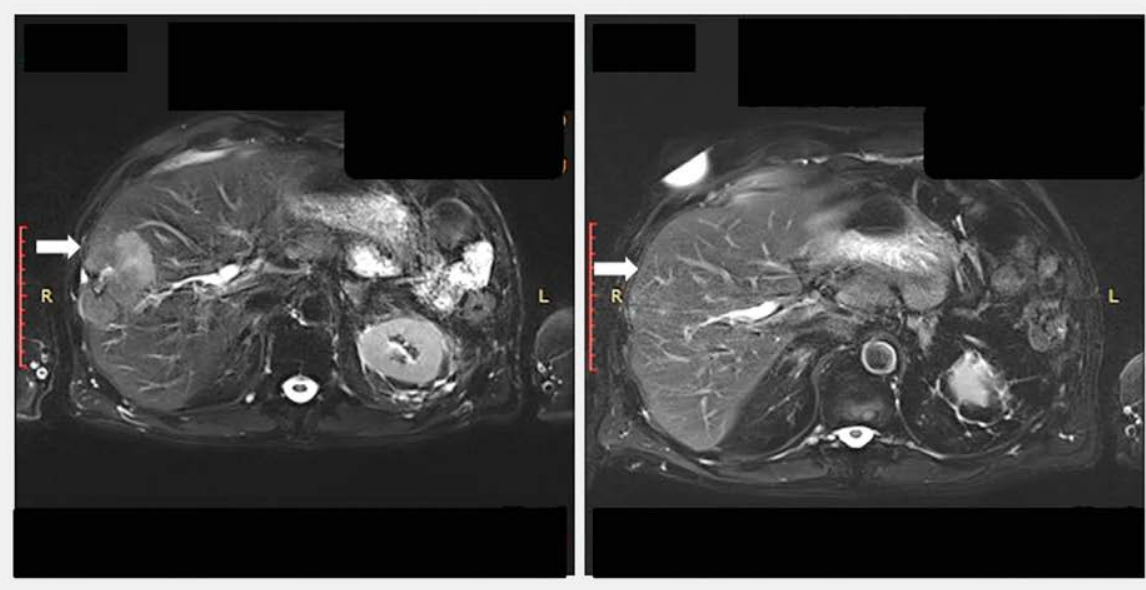

- Fig. 2 Significant liver disease response according to criteria RECIST 1 after one year of treatment with temozolomide-capecitabine. 
sponse rates to treatment with PPRT compared to those with positive FDG-PET/CT $[47,48]$. Thus, a scintigraphic assessment estimating both SSTR expression and glucose metabolism may be required in order to identify patients with more aggressive FDG-positive NENs as these patients may benefit from combination treatment with capecitabine and PRRT $[49,50]$.

In most cases, adverse events associated with PRRT are mild and transient. They include nausea, vomiting, myelosuppression and kidney failure. Co-administration of amino acids is recommended for kidney protection. Rarely, myelodysplastic syndrome and leukaemia have been reported [51-53].

\section{Interferon Alpha (IFN- $\alpha$ )}

IFN- $\alpha$ was initially introduced in 1980 s as a treatment of advanced small bowel NENs and has been associated with control of the symptoms and tumour stabilization [54, 55]. However, a randomized multicentre study showed that SSAs, IFN- $\alpha$, or their combination had comparable antiproliferative effects in the treatment of metastatic neuroendocrine GEP-NENs. However, IFN- $\alpha$ was associated with significant adverse events such as fatigue, fever, liver toxicity, bone marrow suppression and autoimmune disorders leading occasionally to treatment discontinuation $[55,56]$. Thus, due to poor tolerability, IFN- $\alpha$ is currently rarely recommended and its use is restricted in patients with refractory carcinoid syndrome as an add-on treatment to SSAs $[57,58]$.

\section{Immunotherapy}

Immune checkpoint inhibitors have been shown to be effective in several cancer types such as melanoma and lung carcinoma as antibodies against programmed death receptor 1 (PD-1) and programmed death ligand 1 (PD-L1) enhance T-cell antitumour activity. Recent studies have reported PD-L1 expression in GEP-NENs whereas a significant correlation between PD-L1 expression and tumour grade has been observed [59]. Currently there is only limited experience of immunotherapy in NENs but recent case series report promising results while there are multiple ongoing phase II trials that study the efficacy of immune checkpoint inhibitors in NENs [59].

\section{Management of secretory syndromes}

A significant number of NENs secrete biologically active substances and result to development of secretory syndromes. The treatment of patients with functional symptoms should aim primarily in reducing the tumour load either with radical resection or with debulking techniques. However, pre- and perioperative medical treatment is usually necessary while it may also be required in a palliative setting $[60,61]$. When surgery or the cytoreductive techniques fail to control symptoms, medical treatment is utilised aiming at counteracting the effects of the secretory component and/ or control of tumour mass.

Long acting SSAs are considered the most effective option for symptomatic control in patients with several functioning tumours albeit without substantial effect on tumour load [16]. However, an escape phenomenon or tachyphylaxis may occur after a few months or years of treatment with SSAs that it is attributed to a probable down-regulation of SSTRs or tumour progression $[5,20]$ Dose escalation of SSAs may be recommended in case of secretory syndrome refractory to standard doses [20]. In case of insulino- mas, SSAs should be used with caution as worsening of hypoglycaemia may be observed due to inhibition of counter-regulatory hormones [62]. Furthermore, treatment with continuous intravenous infusion of octreotide is required in patients with CS before and during any kind of intervention in order to prevent carcinoid crisis [60]. SSAs can also be used for the treatment of other rare syndromes secondary to the secretion of VIP, glucagon and ectopic hormonal secretion that is occasionally encountered in patients with NENs.

In patients with refractory to SSAs carcinoid syndrome, IFN- $\alpha$ is considered a second line option for symptom control [57]. In addition, PRRT is considered an effective way to ameliorate symptoms of CS [63]. Telotristat etiprate is an oral inhibitor of the enzyme tryptophan hydroxylase which is the rate-limiting step in serotonin synthesis. Recent studies have shown that addition of telotristat in patients inadequately controlled with SSAs is associated with significant reduction in the number of bowel movements, 5-hydroxyindoleacetic acid (5-HIAA) levels and flushing episodes while weight gain was observed in some cases [64-66]. However, this agent has no effect on tumour mass. Common side effects of telotristat include nausea, abdominal pain and a low rate of depression. Further investigation is required regarding the assumption that treatment with telotristat may decrease the development of carcinoid heart disease or fibrosis [64,65].

In patients suffering from insulinoma, the primary treatment target is to avoid hypoglycaemic episodes. Frequent small-volume meals enriched in long-acting carbohydrates are recommended while in some cases, especially in metastatic tumours, continuous feeding via a nasogastric or nasoduodenal tube may be required [61]. Diazoxide decreases insulin secretion through inhibition of adenosine triphosphate (ATP)-sensitive potassium channels. It has been observed to be effective in controlling hypoglycaemia, in doses 50-300 mg/day (maximum dose up to $600 \mathrm{mg} /$ day), but it is associated with significant adverse events such as hirsutism, oedema and renal impairment $[5,67]$. In addition, everolimus has been shown to be effective in reducing hypoglycaemia in cases of metastatic insulinomas while there are some reports regarding the hyperglycaemic action of glucocorticoids [67-69]. Zollinger-Ellison syndrome, secondary to hypersecretion of gastrin from a duodenal or pancreatic NEN may occur sporadically or in approximately $25-30 \%$ of cases in the context of multiple endocrine neoplasia type 1 (MEN1) syndrome [5, 70]. Patients should be treated with high dose of a proton pump inhibitor to reduce the acid hypersecretion while add-on treatment with histamine-2 receptor blockers or antacids may also be required [61] ( Fig. 3 ).

\section{Future Perspectives}

The clinical management of NENs is complex and challenging due to the heterogeneity of these tumours while their low incidence makes research efforts difficult. Novel therapies are currently investigated on multiple ongoing clinical trials while current efforts focus on personalized treatment and precision oncology that target specific genetic and protein regulators of neoplasms.

A biomarker based approach is currently under investigation with the intention to inform the clinicians regarding the prognosis and to facilitate the individualized management of patients with NENs [71]. There are multiple studies investigating the role of sev- 


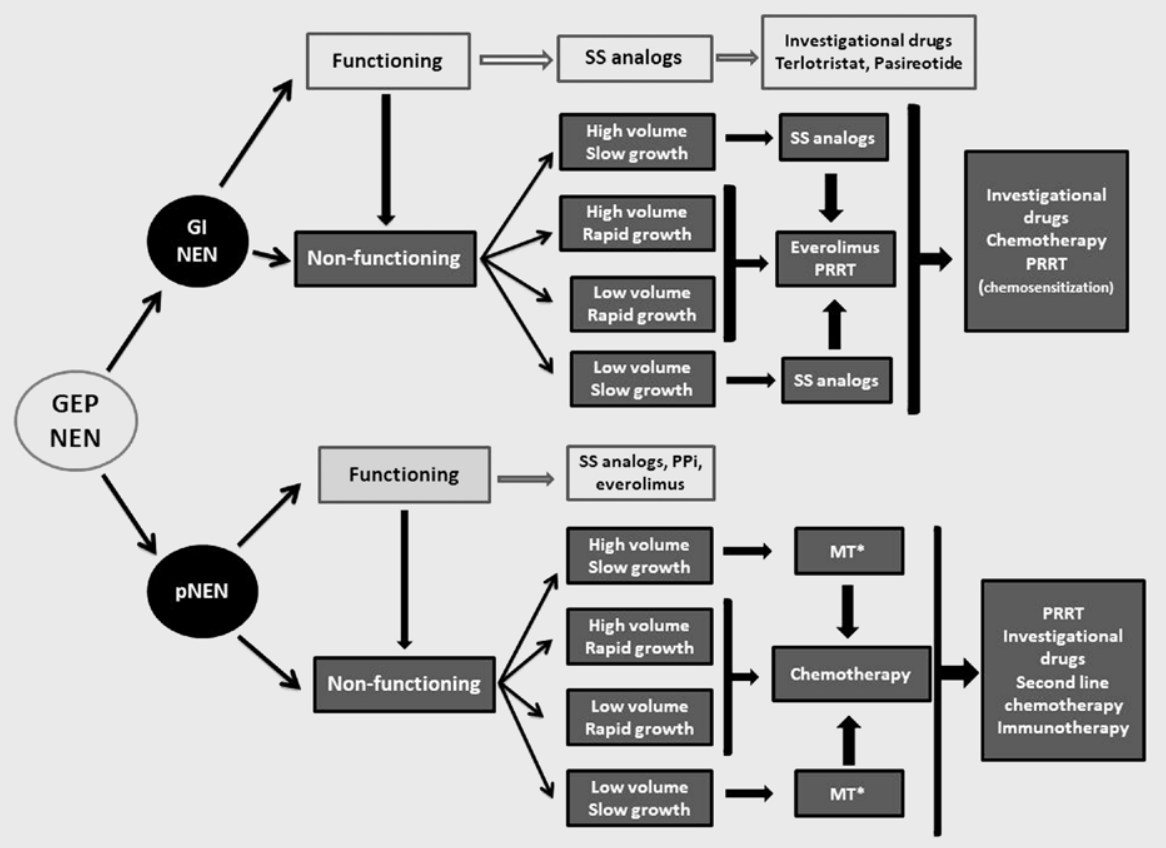

- Fig. 3 Therapeutic algorithm of well differentiated NENs. * Molecular targeted therapy. ${ }^{* *}$ MT: Molecular targeted therapy.

eral biomarkers as well as of genetic and epigenetic alterations, including circulating tumour cells (CTCs), circulating tumour DNA (ctDNA), histone modifications and miRNAs, as prognostic factors and predictors of response to treatment $[71,72]$. A recently developed multigene liquid biopsy (NETest), that is based on transcriptomic evaluation of NENs, has been shown to have numerous clinical applications as it may assess the successful surgical removal of a NEN or predict the aggressive tumour behaviour and the efficacy of SSAs and PRRT [73].

Preclinical in vitro and in vivo models have recently been developed to capture the heterogeneity of human NENs aiming at delineating the biological behaviour of these tumours as well as to investigate the efficacy of new antitumour agents. The patient-derived xenografts (PDX) in mice and zebrafish embryos are considered as the most promising preclinical models for the introduction of personalized medicine [71].

Another issue that needs to be addressed and further studied in the future is the sequential use of treatments available for NENs. A recent retrospective study, comparing different sequences of treatments in patients with well differentiated NENs that received first-line treatment with SSAs, observed no significant difference regarding PFS between patients that received high dose SSAs, everolimus, chemotherapy or PRRT [74]. However, prospective studies are required to further clarify this issue. The rationale of medical treatment taking into consideration factors that could affect the choice of specific treatment for well differentiated GEPNENs is shown in the therapeutic algorithm in > Fig. $\mathbf{3}$.

\section{Conclusion}

NENs are rare tumours that display heterogeneity of biological behaviour and response to treatment. Recent advances regarding the molecular pathways involved in tumour development and new imaging techniques that are associated with the biology of the neoplasms have led to the introduction of new therapeutic modalities such as molecular targeted agents and PRRT that have optimized the treatment of NENs. The management of patients suffering from NENs should be individualized and decisions taken in a multidisciplinary context to improve patients' outcome. Central registration of the patients and response to treatments would be beneficial and would probably ameliorate the management of NENs based on a personalized approach.

\section{Conflict of Interest}

Gregory Kaltsas declare that has received lecture fees from Novo Nordisk and Novartis. All other authors declare that no conflicts of interest exists.

\section{References}

[1] Modlin IM, Oberg K, Chung DC et al. Gastroenteropancreatic neuroendocrine tumours. Lancet Oncol 2008; 9: 61-72

[2] Huguet I, Grossman AB, O'Toole D. Changes in the Epidemiology of Neuroendocrine Tumours. Neuroendocrinology 2017; 104: 105-111

[3] Yao JC, Hassan M, Phan A et al. One hundred years after "carcinoid": Epidemiology of and prognostic factors for neuroendocrine tumors in 35,825 cases in the United States. J Clin Oncol 2008; 26: 3063-3072

[4] Crona J, Norlen O, Antonodimitrakis P et al. Multiple and Secondary Hormone Secretion in Patients With Metastatic Pancreatic Neuroendocrine Tumours. J Clin Endocrinol Metab 2016; 101: 445-452 
[5] Kaltsas GA, Besser GM, Grossman AB. The diagnosis and medical management of advanced neuroendocrine tumors. Endocr Rev 2004; 25: $458-511$

[6] Kloppel G, Rindi G, Perren A et al. The ENETS and AJCC/UICC TNM classifications of the neuroendocrine tumors of the gastrointestinal tract and the pancreas: A statement. Virchows Arch 2010; 456: 595-597

[7] Basturk O, Yang Z, Tang LH et al. The high-grade (WHO G3) pancreatic neuroendocrine tumor category is morphologically and biologically heterogenous and includes both well differentiated and poorly differentiated neoplasms. Am J Surg Pathol 2015; 39: 683-690

[8] Klimstra DS, Kloppell G, La Rosa S et al. Classification of neuroendocrine neoplasms of the digestive system. In: WHO Classification of Tumours: Digestive System Tumours, 5th ed, WHO Classification of Tumours Editorial Board (Ed.), International Agency for Research on Cancer. Lyon. 2019; 16

[9] Tsoli M, Chatzellis E, Koumarianou A et al. Current best practice in the management of neuroendocrine tumors. Ther Adv Endocrinol Metab 2019; 10: 2042018818804698

[10] Kaderli RM, Spanjol M, Kollar A et al. Therapeutic options for neuroendocrine tumors: A systematic review and network meta-analysis. JAMA Oncol 2019; 5: 480-489

[11] Laskaratos FM, Caplin M. Treatment challenges in and outside a network setting: Gastrointestinal neuroendocrine tumours. Eur ] Surg Oncol 2019; 45: 52-59

[12] Oberg KE, Reubi JC, Kwekkeboom D] et al. Role of somatostatins in gastroenteropancreatic neuroendocrine tumor development and therapy. Gastroenterology 2010; 139: 742-53 753 e1

[13] Grozinsky-Glasberg S, Grossman AB, Korbonits M. The role of somatostatin analogues in the treatment of neuroendocrine tumours. Mol Cell Endocrinol 2008; 286: 238-250

[14] de Herder WW, Hofland LJ, van der Lely AJ et al. Somatostatin receptors in gastroentero-pancreatic neuroendocrine tumours. Endocr Relat Cancer 2003; 10: 451-458

[15] Chan JA, Kulke MH. Progress in the treatment of neuroendocrine tumors. Curr Oncol Rep 2009; 11: 193-199

[16] Modlin IM, Pavel M, Kidd M et al. Review article: somatostatin analogues in the treatment of gastroenteropancreatic neuroendocrine (carcinoid) tumours. Aliment Pharmacol Ther 2010; 31: 169-188

[17] Merola E, Panzuto F, Delle Fave G. Antiproliferative effect of somatostatin analogs in advanced gastro-entero-pancreatic neuroendocrine tumors: A systematic review and meta-analysis. Oncotarget 2017; 8: 46624-46634

[18] Rinke A, Muller HH, Schade-Brittinger C et al. Placebo-controlled, double-blind, prospective, randomized study on the effect of octreotide LAR in the control of tumor growth in patients with metastatic neuroendocrine midgut tumors: a report from the PROMID Study Group. J Clin Oncol 2009; 27: 4656-4663

[19] Caplin ME, Pavel M, Cwikla J et al. Lanreotide in metastatic enteropancreatic neuroendocrine tumors. N Engl J Med 2014; 371: 224-233

[20] Strosberg JR, Benson AB, Huynh L et al. Clinical benefits of above-standard dose of octreotide LAR in patients with neuroendocrine tumors for control of carcinoid syndrome symptoms: A multicenter retrospective chart review study. Oncologist 2014; 19: 930-936

[21] Pavel M, Valle JW, Eriksson B et al. ENETS consensus guidelines for the standards of care in neuroendocrine neoplasms: Systemic therapy biotherapy and novel targeted agents. Neuroendocrinology 2017; 105: 266-280

[22] Cives M, Kunz PL, Morse B et al. Phase II clinical trial of pasireotide long-acting repeatable in patients with metastatic neuroendocrine tumors. Endocr Relat Cancer 2015; 22: 1-9
[23] Wolin EM, Jarzab B, Eriksson B et al. Phase III study of pasireotide long-acting release in patients with metastatic neuroendocrine tumors and carcinoid symptoms refractory to available somatostatin analogues. Drug Des Devel Ther 2015; 9: 5075-5086

[24] Alexandraki KI, Daskalakis K, Tsoli M et al. Endocrinological Toxicity Secondary to Treatment of Gastroenteropancreatic Neuroendocrine Neoplasms (GEP-NENs). Trends Endocrinol Metab. 2019; pii: S1043-2760(19)30225-5 . doi: 10.1016/j.tem.2019.11.003 [Epub ahead of print]

[25] Yao JC, Phan A, Chang D et al. Efficacy of RAD001 (everolimus) and octreotide LAR in advanced low- to intermediate-grade neuroendocrine tumors: results of a phase II study. J Clin Oncol 2008; 26 : 4311-4318

[26] Pavel ME, Hainsworth JD, Baudin E et al. Everolimus plus octreotide long-acting repeatable for the treatment of advanced neuroendocrine tumours associated with carcinoid syndrome (RADIANT-2): A randomised, placebo-controlled, phase 3 study. Lancet 2011; 378 : (9808) 2005-2012

[27] Yao JC, Shah MH, Ito T et al. Everolimus for advanced pancreatic neuroendocrine tumors. N Engl J Med 2011; 364: 514-523

[28] Yao JC, Pavel M, Lombard-Bohas C et al. Everolimus for the treatment of advanced pancreatic neuroendocrine tumors: Overall survival and circulating biomarkers from the randomized, phase III RADIANT-3 study. J Clin Oncol 2016; 34: 3906-3913

[29] Pavel M, O'Toole D, Costa F et al. ENETS Consensus Guidelines Update for the Management of Distant Metastatic Disease of Intestinal, Pancreatic, Bronchial Neuroendocrine Neoplasms (NEN) and NEN of Unknown Primary Site. Neuroendocrinology 2016; 103: 172-185

[30] Raymond E, Daham L, Raoul JL et al. Sunitinib malate for the treatment of pancreatic neuroendocrine tumors. N Engl J Med 2011; 364 : 501-513

[31] Phan AT, Halperin DM, Chan JA et al. Pazopanib and depot octreotide in advanced, well-differentiated neuroendocrine tumours: A multicentre, single-group, phase 2 study. Lancet Oncol 2015; 16 : 695-703

[32] Strosberg JR, Cives M, Hwang J et al. A phase II study of axitinib in advanced neuroendocrine tumors. Endocr Relat Cancer 2016; 23 : 411-418

[33] Auernhammer C], Spitzweg C, Angele MK et al. Advanced neuroendocrine tumours of the small intestine and pancreas: clinical developments, controversies, and future strategies. Lancet Diabetes Endocrinol 2018; 6: 404-415

[34] Angelousi A, Kamp K, Kaltsatou M et al. Sequential Everolimus and Sunitinib Treatment in Pancreatic Metastatic Well-Differentiated Neuroendocrine Tumours Resistant to Prior Treatments. Neuroendocrinology 2017; 105: 394-402

[35] Moertel CG, Hanley JA, Johnson LA. Streptozocin alone compared with streptozocin plus fluorouracil in the treatment of advanced islet-cell carcinoma. N Engl J Med 1980; 303: 1189-1194

[36] Moertel CG, Lefkopoulo M, Lipsitz S et al. Streptozocin-doxorubicin, streptozocin-fluorouracil or chlorozotocin in the treatment of advanced islet-cell carcinoma. N Engl J Med 1992; 326: 519-523

[37] Dilz LM, Denecke T, Steffen IG et al. Streptozocin/5-fluorouracil chemotherapy is associated with durable response in patients with advanced pancreatic neuroendocrine tumours. Eur J Cancer 2015; 51: $1253-$ 1262

[38] Heetfeld M., Chougnet CN, Olsen IH et al. Characteristics and treatment of patients with $\mathrm{G} 3$ gastroenteropancreatic neuroendocrine neoplasms. Endocr Relat Cancer 2015; 22: 657-664

[39] Wong MH, Chan D, Lee A et al. Systematic Review and Meta-Analysis on the Role of Chemotherapy in Advanced and Metastatic Neuroendocrine Tumor (NET). PLoS One 2016; 11: e0158140 
[40] Angelousi A, Kaltsas G, Koumarianou A et al. Chemotherapy in NETs: When and how. Rev Endocr Metab Disord 2017; 18: 485-497

[41] Strosberg JR, Fine RL, Choi J et al. First-line chemotherapy with capecitabine and temozolomide in patients with metastatic pancreatic endocrine carcinomas. Cancer 2011; 117: 268-275

[42] Chatzellis E, Angelousi A, Daskalakis K et al. Activity and Safety of Standard and Prolonged Capecitabine/Temozolomide Administration in Patients with Advanced Neuroendocrine Neoplasms. Neuroendocrinology 2019; 109: 333-345

[43] Garcia-Carbonero R, Rinke A, Valle JW et al. ENETS Consensus Guidelines for the Standards of Care in Neuroendocrine Neoplasms. Systemic Therapy 2: Chemotherapy. Neuroendocrinology 2017; 105 : 281-294

[44] Ahlman H, Nilsson O, McNikol AM et al. Poorly-differentiated endocrine carcinomas of midgut and hindgut origin. Neuroendocrinology 2008; 87 : 40-46

[45] Strosberg J, El-Haddad G, Wolin E et al. Phase 3 Trial of (177) Lu-Dotatate for Midgut Neuroendocrine Tumors. N Engl J Med 2017; 376: 125-135

[46] Strosberg J, Wolin E, Chasen B et al. Health-Related Quality of Life in Patients With Progressive Midgut Neuroendocrine Tumors Treated With (177)Lu-Dotatate in the Phase III NETTER-1 Trial. J Clin Oncol 2018; 36: 2578-2584

[47] Severi S, Nanni O, Bodei L et al. Role of 18FDG PET/CT in patients treated with 177Lu-DOTATATE for advanced differentiated neuroendocrine tumours. Eur J Nucl Med Mol Imaging 2013; 40: 881-888

[48] Sansovini M, Severi S, lanniello A et al. Long-term follow-up and role of FDG PET in advanced pancreatic neuroendocrine patients treated with (177)Lu-D OTATATE. Eur J Nucl Med Mol Imaging 2017; 44: 490-499

[49] Claringbold PG, Brayshaw PA, Price RA et al. Phase II study of radiopeptide 177Lu-octreotate and capecitabine therapy of progressive disseminated neuroendocrine tumours. Eur J Nucl Med Mol Imaging 2011; 38: 302-311

[50] Kashyap R, Hofman MS, Michael M et al. Favourable outcomes of (177) Lu-octreotate peptide receptor chemoradionuclide therapy in patients with FDG-avid neuroendocrine tumours. Eur J Nucl Med Mol Imaging 2015; 42: 176-185

[51] Cives M, Strosberg J. Radionuclide Therapy for Neuroendocrine Tumors. Curr Oncol Rep 2017; 19: 9

[52] Bodei L, Kidd M, Paganelli G et al. Long-term tolerability of PRRT in 807 patients with neuroendocrine tumours: the value and limitations of clinical factors. Eur J Nucl Med Mol Imaging 2015; 42: 5-19

[53] Baum RP, Kulkarni H, Singh A et al. Results and adverse events of personalized peptide receptor radionuclide therapy with (90)Yttrium and (177)Lutetium in 1048 patients with neuroendocrine neoplasms. Oncotarget 2018; 9: 16932-16950

[54] Oberg K. Biotherapies for GEP-NETs. Best Pract Res Clin Gastroenterol 2012; 26: 833-841

[55] Oberg K, Norheim I, Lind E et al. Treatment of malignant carcinoid tumors with human leukocyte interferon: long-term results. Cancer Treat Rep 1986; 70: 1297-1304

[56] Faiss S, Pape UF, Bohmig M et al. Prospective, randomized, multicenter trial on the antiproliferative effect of lanreotide, interferon alfa, and their combination for therapy of metastatic neuroendocrine gastroenteropancreatic tumors--the International Lanreotide and Interferon Alfa Study Group. J Clin Oncol 2003; 21: 2689-2696

[57] Oberg K. Interferon in the management of neuroendocrine GEP-tumors: A review. Digestion 2000; 62: (Suppl 1) 92-97
[58] Niederle B, Pape UF, Costa F et al. ENETS Consensus Guidelines Update for Neuroendocrine Neoplasms of the Jejunum and Ileum. Neuroendocrinology 2016; 103: 125-138

[59] Chauhan A, Horn M, Magee G et al. Immune checkpoint inhibitors in neuroendocrine tumors: A single institution experience with review of literature. Oncotarget 2018; 9: 8801-8809

[60] Kaltsas G, Caplin M, Davies P et al. ENETS Consensus Guidelines for the Standards of Care in Neuroendocrine Tumors: Pre- and Perioperative Therapy in Patients with Neuroendocrine Tumors. Neuroendocrinology 2017; 105: 245-254

[61] Dimitriadis GK, Weickert MO, Randeva HS et al. Medical management of secretory syndromes related to gastroenteropancreatic neuroendocrine tumours. Endocr Relat Cancer 2016; 23: R423-R436

[62] Ito T, Igarashi H, Jensen RT. Pancreatic neuroendocrine tumors: clinical features, diagnosis and medical treatment: advances. Best Pract Res Clin Gastroenterol 2012; 26: 737-753

[63] Hofland J, Herrera Martinez AD, Zandee WT et al. Management of carcinoid syndrome: a systematic review and meta-analysis. Endocr Relat Cancer. 2019; pii: ERC-18-0495.R1. doi: 10.1530/ERC-18-0495 [Epub ahead of print]

[64] Kulke MH, Horsch D, Caplin ME et al. Telotristat Ethyl, a Tryptophan Hydroxylase Inhibitor for the Treatment of Carcinoid Syndrome. J Clin Oncol 2017; 35: 14-23

[65] Pavel M, Horsch D, Caplin M et al. Telotristat etiprate for carcinoid syndrome: a single-arm, multicenter trial. ] Clin Endocrinol Metab 2015; 100: 1511-1519

[66] Weickert MO, Kaltsas G, Horsch D et al. Changes in Weight Associated With Telotristat Ethyl in the Treatment of Carcinoid Syndrome. Clin Ther 2018; 40: 952-962

[67] de Herder WW, Schaik E, Kwekkeboom D et al. New therapeutic options for metastatic malignant insulinomas. Clin Endocrinol (Oxf) 2011; 75: 277-284

[68] Kulke MH, Bergsland EK, Yao JC. Glycemic control in patients with insulinoma treated with everolimus. N Engl J Med 2009; 360: 195-197

[69] Novotny J, Janku F, Mares P et al. Symptomatic control of hypoglycaemia with prednisone in refractory metastatic pancreatic insulinoma. Support Care Cancer 2005; 13: 760-762

[70] Jensen RT, Gadiot G, Brandi ML et al. ENETS Consensus Guidelines for the management of patients with digestive neuroendocrine neoplasms: Functional pancreatic endocrine tumor syndromes. Neuroendocrinology 2012; 95: 98-119

[71] Gaudenzi G, Dicitore A, Carra S et al. Management of Endocrine Disease: Precision medicine in neuroendocrine neoplasms: An update on current management and future perspectives. Eur J Endocrinol 2019; 181: R1-R10

[72] Neychev V, Steinberg SM, Cottle-Delisle C et al. Mutation-targeted therapy with sunitinib or everolimus in patients with advanced low-grade or intermediate-grade neuroendocrine tumours of the gastrointestinal tract and pancreas with or without cytoreductive surgery: Protocol for a phase II clinical trial. BMJ Open 2015; 5: 008248

[73] Malczewska A, Kos-Kudla B, Kidd M et al. The clinical applications of a multigene liquid biopsy (NETest) in neuroendocrine tumors. Adv Med Sci 2019; 65: 18-29

[74] Faggiano A, Di Maio S, Mocerino C et al. Therapeutic sequences in patients with grade 1-2 neuroendocrine tumors (NET): An observational multicenter study from the ELIOS group. Endocrine 2019; 66: $417-424$ 